\title{
El sentido de la educación desde lo humano: Apuntes a partir de Martha Nussbaum y Francisco Ruiz Sánchez
}

\author{
The Meaning of Education From a Human Perspective: Remarks From Martha Nussbaum \\ and Francisco Ruiz Sánchez
}

\section{O sentido da educação proveniente do humano: notas de Martha Nussbaum e Francisco Ruiz Sánchez}

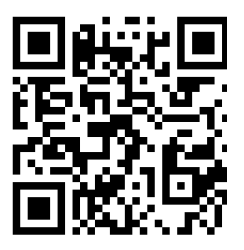

\author{
María Elisa Di Marco \\ Consejo Nacional de Investigaciones Científicas y Técnicas, \\ Centro de Investigaciones Cuyo \\ Mendoza, Argentina \\ mariaelisadimarco@hotmail.com \\ https://orcid.org/0000-0003-4972-9348
}

Recibido • Received • Recebido: 11 / 04 / 2018

Corregido • Revised • Revisado: 02 / 09 / 2019

Aceptado • Accepted • Aprovado: 13 / $11 / 2019$

\begin{abstract}
Resumen: En el presente trabajo, se realiza un análisis del sentido de la educación desde una mirada humana a partir del pensamiento de Martha Nusbaum y Francisco Ruiz Sánchez, en el que se observan algunos puntos en común y algunas diferencias entre los planteos de estas personas autoras. En efecto, en contraposición de las posturas mercantilistas y a favor de una apuesta antropológica, sus estudios insisten en la necesidad de una educación a la medida del ser humano. El estudio hermenéutico que se propone aquí se centra en la teoría de las capacidades de Nussbaum $(2007,2012)$ y la vinculación de esta con el concepto de dignidad humana. Por otra parte, se abordan conceptos fundamentales de la visión antropológica de Ruiz Sánchez (1962a, 1962b, 1978a) como el de la perfectibilidad y la falibilidad, además de otros aspectos que promueven la educación como búsqueda de la plenitud humana. Luego de realizar este análisis, se arriba a la conclusión de que existen numerosos aspectos en común entre las dos visiones revisadas, sin dejar de advertir que también poseen diferencias importantes. No obstante, ambas teorizaciones posibilitan comprender la educación desde una perspectiva humanista, es decir, como un fin en sí misma, pues dota al ser humano de dignidad y plenitud.
\end{abstract}

Palabras claves: Martha Nussbaum; Francisco Ruiz Sánchez; desarrollo humano; capacidad; dignidad humana; educación humanista. 
doi: http://doi.org/10.15359/ree.24-1.15

URL: http://www.una.ac.cr/educare

CORREO: educare@una.cr

\begin{abstract}
This paper analyzes the meaning of education from a humanist perspective developed by Martha Nussbaum and Francisco Ruiz Sánchez. There are some common points and some important differences between the proposals of these authors. In fact, in contrast to the mercantilist perspective and in favor of an anthropological position, both authors insist on the need for a more humanist education, at the measure of the human being. The hermeneutic study proposed here focuses on Nussbaum's theory of capabilities $(2007,2012)$ and its links with the concept of human dignity. On the other hand, this paper presents basic concepts about the anthropological view of Ruiz Sánchez (1962a, 1962b, 1978a) such as perfectibility and fallibility, as well as other aspects that promote education as a search for human fulfillment. After carrying out this analysis, we conclude that there are many aspects in common between the two authors reviewed, but also important differences. However, both theorizations make it possible to understand education from a humanist perspective, that is, as an end in itself, because it endows the human being with dignity and fullness.
\end{abstract}

Keywords: Martha Nussbaum; Francisco Ruiz Sánchez; human development; capacity; human dignity; humanist education.

Resumo: Neste artigo, é feita uma análise do sentido da educação a partir de uma perspectiva humana, com base nos pensamentos de Martha Nusbaum e Francisco Ruiz Sánchez, nos quais são observados alguns pontos em comum e algumas diferenças entre as abordagens dessas pessoas como autoras. De fato, em contraste com as posições mercantilistas e a favor de um compromisso antropológico, seus estudos insistem na necessidade de uma educação adaptada ao ser humano. O estudo hermenêutico proposto aqui enfoca a teoria das capacidades de Nussbaum $(2007,2012)$ e sua ligação com o conceito de dignidade humana. Por outro lado, são abordados conceitos fundamentais da visão antropológica de Ruiz Sánchez (1962a, 1962b, 1978a), como o de perfeição e falibilidade, além de outros aspectos que promovem a educação como busca da realização humana. Após realizar essa análise, a conclusão encontrada é que existem inúmeros aspectos em comum entre as duas visões revisadas, observando que elas também apresentam diferenças importantes. No entanto, ambas teorizações tornam possível entender a educação sob uma perspectiva humanística, isto é, como um fim em si mesmo, porque confere dignidade e plenitude ao ser humano.

Palavras-chave: Martha Nussbaum; Francisco Ruiz Sánchez; desenvolvimento humano; capacidade; dignidade humana; educação humanista.

Por todo el mundo hay personas que se esfuerzan en llevar unas vidas humanamente dignas. Los dirigentes de los países suelen centrarse exclusivamente en el crecimiento económico nacional, pero sus ciudadanos y sus ciudadanas se afanan, mientras tanto, por conseguir algo distinto: unas vidas significativas para sí... (Nussbaum, 2012, p. 19)

\title{
Introducción
}

Repensar y recuperar el sentido de lo humano es una tarea imprescindible en lo que respecta al ámbito educativo. Profundizar en este asunto podría considerarse como el cometido fundamental de una de las intencionalidades presentes tanto en Martha Nussbaum como en Francisco Ruiz Sánchez, ya que desde sus apreciaciones acerca del ser humano puede inferirse un sentido de la educación netamente humanista. 
Así pues, la defensa de la educación humanista y la posibilidad de pensar una educación a la medida del ser humano, que favorezca el desarrollo de su dignidad y plenitud, son elementos que invitan a replantear el centro de las propuestas políticas-pedagógicas actuales. El problema puede interpretarse desde interrogantes profundos como los siguientes: ¿Qué entendemos por educación? ¿Más educación en sentido cuantitativo representa realmente un avance en la formación integral del ser humano? ¿Qué implica una educación a la medida del ser humano? Sin embargo, varios de los planteos actuales se reducen a una mejora educativa ligada fuertemente a lo económico, que olvidan muchas veces al ser humano mismo (y su dignidad) reduciéndolo a un ser meramente material.

Esta crisis silenciosa señalada por Nussbaum (2010) es uno de los mayores desafíos que presenta la educación contemporánea, no solo para aquellos países del llamado primer mundo que han alcanzado cierto estatus económico y siguen direccionando la educación hacia el acrecentamiento de sus riquezas, sino también para aquellos en vías de desarrollo que buscan replicar un modelo por competencias basado en lo económico. Pues, ambos parecen errar en el mismo sentido, a saber, en descuidar en qué radica la educación misma, ordenada a la plenificación integral del ser humano.

En esta discusión se introducen dos miradas: la de Martha Nussbaum y la de Francisco Ruiz Sánchez.

Francisco RuizSánchez (1929-1982)fue profesor einvestigadormendocino de Argentina, quien llevó a cabo un análisis de diversos aspectos educativos en los que justifica, a partir de los conceptos de perfectibilidad y falibilidad, la necesidad de una interpretación adecuada del ser humano al abordar las consideraciones educativas. Su pensamiento puede comprenderse como un paso más y una fundamentación coherente de la plenitud del ser humano como finalidad pedagógica.

Martha Craven Nussbaum es estadounidense nacida en 1947, Doctora en Derecho y Ética egresada de Harvard en 1975. Junto con el economista Amartya Sen trabajó en temas relacionados con el desarrollo y la ética. Desempeñó la docencia en universidades como Brown, Harvard y Oxford. En el año 2012 fue galardonada con el premio Príncipe de Asturias de Ciencias Sociales, entre otros antecedentes científicos y académicos de prestigio (Nussbaum, 2010; 2012).

Ambas posiciones parecen dirigirse el mismo objetivo, si bien vale aclarar, parten desde distintos supuestos y planteos. Más allá de estas diferencias sustanciales que se verán reflejadas en ambos pensamiento, es interesante rescatar que vuelven la mirada sobre una misma idea: la de otorgar un sentido eminentemente humano a la educación.

En esta dirección, el objetivo de este trabajo es realizar un análisis hermenéutico que resalte las diferencias y exponga algunos elementos que conforman un concepto más amplio de educación, a partir del análisis de elementos centrales tanto del pensamiento de Nussbaum, como de Ruiz Sánchez. 
doi: http://doi.org/10.15359/ree.24-1.15

URL: http://www.una.ac.cr/educare

CORREO: educare@una.cr

Así pues, una vez referida la metodología de investigación se presenta la teoría de las capacidades de Martha Nussbaum y la importancia de estas en el proceso de desarrollo humano a favor de la dignidad del ser humano. Asimismo, sobre el pedagogo argentino se expone su propuesta acerca de los fundamentos antropológicos, éticos y sociales de la educación, en los que hacen su aparición los conceptos de perfectibilidad y falibilidad, claves para comprender su visión educativa como plenitud dinámica. Posteriormente, se establecen algunas comparaciones sobre el análisis efectuado a partir de un diálogo entre ambos pensamientos y se concluye al respecto.

\section{Metodología de la investigación}

La metodología por emplear es eminentemente cualitativa, a saber, la hermenéutica de lectura, análisis e interpretación crítica de las distintas fuentes bibliográficas (Gadamer, 2015; García-Amilburu, 2008). Debido a que "los enunciados que integran el escrito están constituidos por un conjunto de signos o palabras que expresan un contenido que necesita ser interpretado" (Bicocca, 2008, p. 104), se apunta a realizar una lectura cuidadosa y analítica de los diversos escritos para interpretar las categorías susceptibles de ser analizadas y los supuestos teóricos subyacentes.

Como señala Bicocca (2008): “... el texto encierra en sí una significación o un sentido que es necesario desentrañar y esclarecer" (p. 104). Se trata de mostrar el significado de lo que expresan, su sentido más profundo, desde dónde lo dicen y en relación con qué teorías. De este modo, se favorece la intertextualidad con el fin de develar e interpretar el sentido de los textos de la autora y el autor que se abordan en estudio.

Como explica García-Amilburu (2008):

El interés principal de la hermenéutica consiste en investigar de qué manera y bajo qué condiciones, tiene sentido algo que ha sido dicho o hecho en el pasado -o en la actualidad-, de modo que pueda ser reconocido como un texto dentro de una tradición [cursivas añadidas]. (p. 107)

Teniendo en cuenta estas consideraciones, se lleva a cabo una interpretación de lo que expresan los textos, favoreciendo la intertextualidad en relación con el sentido que le otorgan a la educación desde una visión que toma en consideración diversos factores, no solo el económico. En otras palabras, se analizan en sus obras cómo se presenta en cada quien la mirada del ser humano que permite, posteriormente, deducir el sentido que le otorgan a la educación. De allí se interpretan algunos puntos en común y algunas diferencias entre sus planteos. Esto nos va a permitir descubrir que existen numerosos aspectos en común en las dos miradas y diferencias importantes; pero que, en consecuencia, ambas teorizaciones posibilitan comprender la educación desde una perspectiva humanista, que dota al ser humano de dignidad y plenitud. 


\section{El sentido de lo humano en Nussbaum: Análisis a favor de una vida digna}

Martha Nussbaum, como señala Bicocca (2015), nació en Nueva York y desarrolló sus estudios de teatro y lenguas clásicas en la Universidad de Nueva York (1969), luego se graduó en Filosofía por la Universidad de Harvard (1972); una vez graduada, enseñó filosofía y letras clásicas en Harvard hasta principios de los ochenta, luego se trasladó a la Universidad de Brown (Providence, Rothe Island) y actualmente se desempeña como profesora en la Facultad de Derecho de la Universidad de Chicago. Debido a la calidad de sus escritos ha sido reconocida con premios y títulos honoríicos internacionalmente.

Como señala Nussbaum (2010, siguiendo a Dewey, 1916), el logro viene a equivaler a la clase de cosas que una máquina bien planeada puede hacer mejor que un ser humano, y el efecto principal de la educación -la construcción de una vida plena de significación- queda al margen.

Desde esta plataforma, resulta importante plantearse en la actualidad hacia dónde está tendiendo la educación, cuál es su finalidad, cuál es la motivación última que la anima (entre otros tantos interrogantes), a fin de propender a una reflexión antropológica seria que confiera al ser humano centralidad en los planteos educativos.

Con la intención de defender una educación verdaderamente humanista, Nussbaum (2010) hace una fuerte crítica a aquellos planteamientos que alimentan una "crisis silenciosa" debido a la sed de ingresos, dejando de lado un amplio abanico de aptitudes que favorecen la vida en democracia. De este modo, expresa la profunda crisis mundial que existe en materia educativa, de la cual no escapan los países de Latinoamérica. Con base en estos planteos, en Sin fines de lucro. Por qué la democracia necesita de las humanidades (Nussbaum, 2010) la filósofa critica:

En casi todas las naciones del mundo se están erradicando las materias y las carreras relacionadas con las artes y las humanidades, [por concebirlas] ... como ornamentos inútiles por quienes definen las políticas estatales en un momento en que [los gobernantes de] las naciones [juzgan que] deben eliminar todo lo que no tenga ninguna utilidad para ser competitivas en el mercado global. (Nussbaum, 2010, p. 20)

Además, como explica en otra de sus obras, "tanto la filosofía como la literatura, en cuanto búsquedas de la verdad, de respuestas frente a un núcleo de preguntas vitales, recurren a una estructura formal que necesariamente es una actitud frente a la vida" (Nussbaum 1995, p. 106).

O'Brien (2010, p. 13) "desmitifica la idea de que la educación sea principalmente una herramienta para el crecimiento económico y argumenta que ... [esto] no supone de manera necesaria una mejora en la calidad de vida. El descuido y el desprecio de las artes y las humanidades generan un peligro ... [en este sentido] para la salud de nuestras democracias". La mentalidad mercantilista que ha imperado en las políticas educativas mundiales como sostiene Bicocca (2015): 
doi: http://doi.org/10.15359/ree.24-1.15

URL: http://www.una.ac.cr/educare

CORREO: educare@una.cr

... por una parte, ha conducido a centrarla más en los procesos y en los factores que los facilitan que en sus fines, hasta el punto de olvidar su sentido y razón de ser. Por otra, ha generado una distorsión del quehacer educativo, pensando que consiste en modelar individuos bien adaptados a la maquinaria socio-político-productiva. (p. 29)

Estudios como los de Pring (2016) se hacen eco, también, de estas demandas. En efecto, este denuncia, en sus trabajos compilados por García-Amilburu (2008), la mentalidad mercantilista que penetra y controla los sistemas educativos actuales cambiando sus valores, las formas de relacionarse de los sujetos participantes involucrados y hasta su mismo lenguaje. En tal dirección, Pring (2016) señala:

No es extraño, por tanto, que la Ética -y la Filosofía en general- ya no se consideren materias necesarias para la preparación de los futuros profesores, porque no se espera que éstos [sic] se impliquen en discusiones acerca de los fines de la educación. (p. 31)

Como explica Gómez (2013):

Nussbaum ha coincidido con Amartya Sen en plantear un nuevo enfoque del desarrollo humano, el cual tuvo su impacto en el informe lanzado por el Programa de las Naciones Unidas para el Desarrollo (PNUD) desde 1990, en donde se sitúa a las capacidades humanas como centro de evaluación alternativa al enfoque clásico de corte utilitarista. (pp. 20-21)

En Crear capacidades, la autora expone este enfoque al que entiende como "una aproximación particular a la evaluación de la calidad de vida y a la teorización sobre la justicia social básica" (Nussbaum, 2012, p. 38). Tal enfoque se pregunta por las oportunidades disponibles en cada ser humano, se centra en la libertad del sujeto y va un paso más allá de la dependencia económica que establecían las demás perspectivas. Desde este marco, esta teoría especifica algunas condiciones necesarias para que una sociedad sea mínimamente justa (Nussbaum, 2007, p. 163). Por consiguiente, estas capacidades se convierten así en metas políticas (Monereo, 2015).

Las capacidades responden a la pregunta: "QQué es capaz de hacer y de ser esta persona?" (Nussbaum, 2012, p. 40). Asimismo, vienen a ser una especie de libertad sustantiva de alcanzar combinaciones alternativas de funcionamiento personal y social. En palabras textuales de la autora: "no son simples habilidades residentes en el interior de una persona, sino que incluyen también las libertades o las oportunidades creadas por la combinación entre esas facultades personales y el entorno político, social y económico" (Nussbaum, 2012, p. 40). De aquí se desprende la noción de "capacidades combinadas" que propone la autora. A su vez, otra distinción alude a la conceptualización de las "capacidades básicas", que son "las facultades innatas de la persona que hacen posible su posterior desarrollo y formación" (Nussbaum, 2012). 
Por otra parte, a diferencia de Sen (2004) que no parece partidario de una lista única de capacidades, Nussbaum presenta una enumeración de las capacidades humanas que debe propiciar la educación (Gough, 2007-2008). Las capacidades centrales que describe son: vida, salud física, integridad física, sentidos, imaginación y pensamiento, emociones, razón práctica, afiliación, relación con otras especies, juego y control del propio entorno (Nussbaum, 2012). Entre ellas, tienen especial atención en su propuesta las capacidades de afiliación y de razón práctica, que desempeñan un papel arquitectónico y mantienen al conjunto de las capacidades (Nussbaum, 1992, 2000). Respecto de la razón práctica, la autora enfatiza su finalidad de permitir formarse una concepción del bien y reflexionar críticamente sobre los propios planes de vida (Nussbaum, 2012).

Lo cierto es que la propuesta de Nussbaum instaura el debate acerca de la necesidad de una educación humanista, esto es, a la medida del ser humano, mediante el desarrollo de estas capacidades, en la cual el concepto de dignidad, o más bien, de vida digna se halle presente. En definitiva:

No necesitamos ganarnos el respeto de los demás siendo productivos. Podemos basar nuestra apelación en la dignidad misma de nuestras necesidades humanas. La sociedad está unida por un amplio abanico de afectos y compromisos, sólo algunos de los cuales tienen que ver con la productividad. La productividad es necesaria, e incluso buena; pero no es la finalidad principal de la vida social. (Nussbaum, 2007, pp. 167-168)

Ahora bien, por otro lado, la misma autora se pregunta sobre la relación que guarda su concepción de bien con la dignidad humana (Nussbaum, 2007).

Aquí, ciertamente, conviene distanciarse del carácter instrumental de las capacidades y aseverar que son formas de hacer efectiva una vida con dignidad humana, mediante las cuales cabe interrogarse sobre estas áreas de vivencia, es decir, sobre cuál sería la capacidad misma y las actividades compatibles con la dignidad humana. La autora responde a este planteo señalando que la dignidad no es algo que pueda definirse de forma previa a las capacidades, sino que se encuentra imbricada en ellas, en su misma definición (Nussbaum, 2007).

Es clara en Nussbaum la idea de que el Estado es el principal encargado de hacer posible el desarrollo de las capacidades, que se produce a través de la combinación de las habilidades personales -capacidades básicas- y las posibilidades que ofrece el entorno -capacidades combinadas-. Esto, ciertamente, favorece el desarrollo de la dignidad humana.

No obstante, es puesta en el funcionamiento la capacidad como se realiza activamente. Como bien explica la autora: "la noción de funcionamiento sirve de punto de destino final para la noción de capacidad" (Nussbaum, 2012, p. 44). Aunque conviene aclarar con la autora que "las capacidades tienen también valor en sí mismas, entendidas como ámbitos de libertad y elección. Promover capacidades es promover áreas de libertad ..." (Nussbaum, 2012, p. 45). 
doi: http://doi.org/10.15359/ree.24-1.15

URL: http://www.una.ac.cr/educare

CORREO: educare@una.cr

Empero, el planteo de la autora estadounidense entra en una especie de encrucijada en el funcionamiento al tratar de develar el hecho de hacer cosas que puedan destruir alguna de las capacidades, como por ejemplo el caso de las drogas (Nussbaum, 2012). Lo cierto es que Nussbaum (2012) se presenta al respecto con cierta flexibilidad y deja en manos del proceso político la posible regulación de estos casos. Como señala Nussbaum:

El enfoque de las capacidades no es una teoría sobre lo que la naturaleza humana es o no es, ni interpreta normas a partir de la naturaleza humana innata ... se pregunta qué cosas, de entre las muchas para las que los seres humanos pueden desarrollar una capacidad de desempeño, son aquellas que una sociedad como mínimo aceptable de justicia se esforzará por nutrir y apoyar ... (Nussbaum, 2012, p. 48)

En este sentido, puede postularse un carácter educativo del Estado como agente primordial propulsor de las capacidades humanas. En el pensamiento de Nussbaum es, de alguna manera, el Estado el que tiene el deber de impulsar el desarrollo de estas capacidades $y$, de este modo, ofrecer oportunidades del despliegue de la dignidad humana. Asimismo, como ha quedado demostrado, no hay en el pensamiento de la autora una lectura objetiva del orden natural -como sí se podrá observar en Ruiz Sánchez- sino una circularidad argumentativa, pues afirma que es la sociedad política la que debe posibilitar el desarrollo de las capacidades; pero, a su vez, es esta sociedad la que de algún modo dictamina el valor y funcionamiento de estas. En esta dirección, como la misma autora asevera:

El enfoque de las capacidades se articula en términos de la idea rawlsiana del liberalismo político; es decir, la teoría de los derechos es vista como una descripción parcial del bien, para fines políticos, que los ciudadanos pueden vincular a diferentes concepciones comprehensivas del bien. El enfoque se articula únicamente, o al menos así lo esperamos, en términos de ideas éticas independientes, sin pasar por ninguna doctrina metafísica y epistemológica (como la doctrina del alma, de la revelación, o la negación de ambas) que pudiera dividir a los ciudadanos en virtud de su religión o de su doctrina ética comprehensiva. Esperamos, pues, que esta concepción pueda ser objeto de un consenso entrecruzado entre unos ciudadanos que mantienen por lo demás diferentes doctrinas comprehensivas. (Nussbaum, 2007, p. 170)

Por consiguiente, es claro que la autora sostiene la imposibilidad de la lectura de la naturaleza, esto es, la metafísica, y por tanto, en pos de un acuerdo global, admite una cierta subjetividad en el planteo de la fundamentación del enfoque educativo que propone. También, se debe tener presente que el gran objetivo que orienta su indagación es lograr que todos los seres humanos "deberían superar un cierto nivel umbral de capacidad combinada, entendiendo ese deber no como un funcionamiento obligado, sino como una libertad sustancial para elegir y actuar" (Nussbaum, 2012, p. 44).

8

Los artículos de la Revista Electrónica Educare del Centro de Investigación y Docencia en Educación de la Universidad Nacional, Costa Rica, se comparten bajo términos de la Licencia Creative Commons: Reconocimiento, NNo Comercial, Sin Obra Derivada 3.0 Costa Rica. Las autorizaciones adicionales a las aquí delimitadas se pueden obtener en el correo: educare@una.cr 
La intención de Nussbaum es valorable, aunque resulta problemática, ya que intenta definir el umbral mencionado a través de una especie de acuerdo sin remitir a una fundamentación objetiva, como es, por ejemplo, la perspectiva natural, incluso cuando este proceder podría derivar en la negación del mismo concepto de dignidad humana al cual pretende tender con el desarrollo de las capacidades. No obstante, se puede reconocer en su teoría la preocupación por un mínimo de desarrollo que nadie se atrevería a objetar.

Lo cierto es que el planteo de Nussbaum trasciende las miras meramente económicas y permite pensar en otras dimensiones del ser humano, sin dejar de reconocer que el sustento que confiere a las capacidades, por tender a cierta flexibilidad desde la determinación del umbral mínimo y la regulación de la libertad, puede tornarse endeble y un tanto confuso. A su posición podría aplicársele la afirmación de McCarthy (1992): “El problema práctico de la vida virtuosa de los ciudadanos de la polis, se transformó en el problema técnico de cómo regular la interacción social para asegurar el orden y el bienestar de los ciudadanos del Estado" (p. 22).

Así pues, la propuesta filosófica de Martha Nussbaum, de manera aproximada se puede considerar como lo propone Arjona (2013, p. 149):

Un intento por recuperar la antigua pregunta por la vida buena y la forja del carácter, sin perder de vista la relevancia y el refinamiento que siglos del pensamiento liberal han dado a la perspectiva de la justicia, cuya pertinencia se conserva en el mundo globalizado que habitamos, en el que coexisten diversas concepciones comprehensivas del bien.

En suma, el planteo de Nussbaum identifica una idea del ser humano como ser libre y digno (Murillo y Hernández, 2011), y es a partir del desarrollo de las capacidades que puede repensarse el sentido de la educación humanista, más allá de las preocupaciones económicas actuales. A continuación, se presenta otra perspectiva educativa que también centra su mirada en lo humano: la de Ruiz Sánchez. En su teorización el concepto de plenitud aboga por una educación humanista.

\section{El sentido de lo humano en Francisco Ruiz Sánchez. La educación como plenitud humana}

En oposición a los planteos mercantilistas en materia educativa, Francisco Ruiz Sánchez han teorizado, al igual que Nussbaum, sobre la posibilidad y necesidad de una visión educativa que profundice acerca del sentido humanístico de la educación.

El profesor e investigador Francisco Ruiz Sánchez (1929-1982) vivió en la provincia de Mendoza de Argentina. A partir de los años setenta, desempeñó su tarea docente en distintas universidades de la región de Cuyo como profesor de filosofía de la educación y pedagogía, entre otras disciplinas académicas. Su actividad como docente universitario fue realizada en la Universidad Nacional de Cuyo, la Universidad Católica de Cuyo y la Universidad Católica Argentina. 
doi: http://doi.org/10.15359/ree.24-1.15

URL: http://www.una.ac.cr/educare

CORREO: educare@una.cr

Ruiz Sánchez ha legado en sus escritos algunas consideraciones sobre aspectos como los fundamentos de la educación, su fin, el concepto de educación y la educabilidad, entre otras categorías que -desde los aportes de la filosofía- pueden fundamentar la reflexión educativa. Entre sus escritos más representativos se encuentra Fundamentos y fines de la educación cuya primera edición se remonta a 1978.

El autor explica, de forma coherente, los fundamentos y los fines que sostienen el hecho educativo, más allá de la consideración del contexto y de las particularidades del sujeto. De este modo, la obra del autor reflexiona sobre la realidad -en este caso la educativa- desde sus fundamentos últimos, al modo en que lo hace el filósofo de la educación y llega hasta el fondo en sus argumentaciones en favor de cimentar el hecho educativo, establecer un concepto de educación y de finalidad educativa.

Conviene aclarar que es imposible elaborar un análisis minucioso de su pensamiento, ya que extendería los límites de este trabajo, pero se deja al menos presentados -al igual que con la autora estadounidense- algunos elementos para repensar la necesidad de una educación humanista desde el planteo de la plenificación del ser humano.

El pensamiento del autor podría dialogar con algunos aspectos ya planteados en la propuesta de Nussbaum a través de las siguientes preguntas: ¿Qué implica que el ser humano viva en dignidad, en plenitud? ¿Qué elementos sustentan un modelo educativo humanista 0 centrado en el ser humano? ¿Qué significa que la educación adquiera un sentido para sí misma y no en función de propuestas mercantilistas?

Es importante remarcar que Ruiz Sánchez no hace una defensa de la educación humanista desde un planteamiento curricular como se puede entender en gran parte el planteo de Nussbaum (2010) en Sin fines de lucro. Por quéla democracia necesita de las humanidades tampoco el autor propone su teoría en función de mejorar las políticas públicas en orden a la justicia social, como también proponía la autora en Crear capacidades (Nussbaum, 2012); sino que, más bien, su pensamiento radica en una mirada antropológica, social y ética de la educación que permite concluir en una definición del concepto de educación y el establecimiento de su finalidad netamente humanista al modo en que discurre la filosofía.

La visión antropológica es el fundamento primero en el pensamiento de Francisco Ruiz Sánchez. En sus diversas obras puede advertirse este abordaje de los temas vinculados con su propuesta sobre los fines de la educación que pone de manifiesto en los siguientes escritos: El concepto del hombre y la educación (Ruiz Sánchez, 1962b), Acerca de la educabilidad (Ruiz Sánchez, 1972a), Pedagogía (Ruiz Sánchez, 1972b), Esquema tentativo para una estructuración de la temática fundamental de la Pedagogía (Ruiz Sánchez, 1973a), Los fines de la educación (Ruiz Sánchez, 1973b), Fundamentos y fines de la educación (Ruiz Sánchez, 1978a), Introducción a los fundamentos antropológicos de la educación (Ruiz Sánchez, 1978b), Posibilidad de considerar la

María Elisa Di Marco

Los artículos de la Revista Electrónica Educare del Centro de Investigación y Docencia en Educación de la Universidad Nacional, Costa Rica, se comparten bajo términos de la Licencia Creative Commons: Reconocimiento, № Comercial, Sin Obra Derivada 3.0 Costa Rica. Las autorizaciones adicionales a las aquí delimitadas se pueden obtener en el correo: educare@una.cr 
educación como un arte (Ruiz Sánchez, 1978c), El hombre ¿actor o juguete de la historia? (Ruiz Sánchez, 1978d), La contaminación de la educación (Ruiz Sánchez, 1979), entre otros. La obra del autor se encuentra aún sujeta a revisión, por lo que uno de los mayores desafíos, para los grupos especialistas en su pensamiento, es establecer su corpus total, publicado y no publicado.

En El concepto del hombre y la educación, Ruiz Sánchez (1962b) sostiene que en el ser humano, como en todo ser vivo, existe un proceso de maduración de su naturaleza. Es indudable que la educación hace que ese proceso sea realmente un proceso de perfeccionamiento, en el cual la naturaleza humana impone su línea y sus límites. En este sentido, Ruiz Sánchez (1962b) recrea las categorías clásicas de la pedagogía realista, así pues: “La maduración cualitativa en que consiste el proceso, no es otra cosa que la actualización de las posibilidades de perfección (perfectibilidad)" (p. 66). Puede el lector o lectora suspicaz encontrar cierta similitud con el concepto de capacidades; aunque conviene advertir que en Ruiz Sánchez la naturaleza es la que, como se dijo, impone su líneas y límites.

Otra característica antropológica en el pensamiento del autor mendocino es lo que denomina "falibilidad". Ruiz Sánchez, parte de la constatación del carácter paradojalmente perfectible del ser humano. Por un lado, la humanidad implica imperfección, cierta falibilidad que conlleva; pero, posibilita, por otro lado, que el mismo ser humano pueda plenificarse, es decir, abrirse a los caminos de la perfectibilidad (Ruiz Sánchez, 1962b), como él mismo asegura:

También el hombre imperfecto, pobre de la humanidad que debiera poseer, busca, exige perfecciones propias que tiene que encarnar, hacer suyas, poseer ... . Necesita "su" perfección de hombre ... una tarea o una concepción educativa implica un conocimiento cierto y adecuado del hombre, de sus fines objetivos (últimos e intermedios) y de su fin último subjetivo.

Un error teórico en la concepción del hombre y de sus fines, tiene consecuencias prácticas desastrosas, pues encarrila la tarea de la formación del hombre sobre supuestos falsos. (Ruiz Sánchez, 1962b, p. 68)

Esto habilita hacer una pregunta, en el marco de esta especie de diálogo entre las personas autoras: ¿Qué sentido tendría el desarrollo de capacidades que propone Nussbaum, si se dieran estas por supuestas y el ser humano solo con su sola existencia las pudiese desarrollar? Ciertamente, para que haya un desarrollo de las capacidades no basta con la sola existencia. El desafío radica en superar la falibilidad e indigencia con que se nace y que ambos pensamientos observan, a su modo, en el mismo ser humano y en la sociedad. Dando un paso más se puede afirmar que hay algo en el mismo ser humano que posibilita tal desarrollo: las capacidades -en términos de Nussbaum- y la perfectibilidad -en términos de Ruiz Sánchez-. No obstante, en ambos idearios, el fin que conceden a esta idea es diverso. 
doi: http://doi.org/10.15359/ree.24-1.15

URL: http://www.una.ac.cr/educare

CORREO: educare@una.cr

A este respecto, puede hacerse un paralelo entre la falibilidad que señala Ruiz Sánchez y la indefensión que marca Nussbaum (2010). De hecho, la autora al tratar en el capítulo tres de Sin fines de lucro. Por qué la democracia necesita de las humanidades (Nussbaum, 2010) acerca de educar la ciudadanía: los sentimientos morales (y antimorales), se pregunta sobre aquello que en la vida humana dificulta la convivencia democrática. Responde que tiene que ver con un relato "sui generis que implica nuestra combinación de competencia con indefensión, nuestra relación problemática con la impotencia, la mortalidad y la finitud" (Nussbaum, 2010, p. 55).

El mérito de Ruiz Sánchez radica en intentar una interpretación lo más exacta posible del ser humano, que evite reduccionismos. Así, no se limita a describir casos o narrar historias, sino que va al meollo de la cuestión y analiza si puede haber un fin de la educación común a todos los seres humanos a partir de una minuciosa consideración antropológica. Para llevar a cabo su análisis, parte de supuestos firmes que sintetiza en Fundamentos y fines de la educación (Ruiz Sánchez, 1978a). Tales supuestos antropológicos están impregnados de una visión clásica de la antropología filosófica, que el autor recrea. En este sentido, elabora trece notas distintivas del ser humano, a saber: como persona, ser dinámico, ser con interioridad, capaz de autoconducción, capaz de elegir, capaz de ser libre, como pluralidad de tendencias, con multiplicidad de dimensiones, ser dependiente, ser condicionado, ser falible, ser histórico y ser religado (Ruiz Sánchez, 1978a).

Lo interesante del planteo del autor es que, sin renunciar a su tradición de tinte clásico, presenta al ser humano en su dimensión esencial retomando el planteo del concepto Boeciano de persona, pero a su vez, reconociendo aspectos irrenunciables del ser humano en los cuales se desenvuelve su existencia, tales como: la dinamicidad, la dependencia, historicidad, religación, etc. aspectos que, en suma, son considerados dentro de la educabilidad y que, por lo tanto, convienen en una consideración educativa humanista. Aquí se distingue su notable mérito en cuanto a la concepción del sujeto, que se comprende -desde esta perspectiva-como una totalidad individual.

Los fundamentos sociales constituyen el segundo pilar de los fundamentos educativos, pues el ser humano es una "parte" de ese todo moral que es la sociedad cuyas partes están relacionadas entre sí en función de un fin, y ese fin es el bien del que todas las personas participan, que es el bien común (Ruiz Sánchez, 1978a). Para el autor, el ser humano es parte de todos reales (familia, club, comunidad religiosa, nación, etc.):

De esta manera el hombre, a la vez que un "todo" individual, se halla inscripto, simultáneamente, con su vida, en varias sociedades de las que es miembro. Por ende, está relacionado con los otros miembros, directa o indirectamente, y con el bien común de cada sociedad que da sentido a aquellas relaciones. Esto porque una "parte" en un todo, tiene -o debe tener- relaciones de congruencia con las otras partes (relación parte-parte) y en función del todo (relación parte-todo). (Ruiz Sánchez, 1978a, p. 203) 
Puede interpretarse aquí que se está ante un punto de encuentro. En efecto, a partir del análisis de estas dos miradas podemos afirmar que Ruiz Sánchez reconoce la relación que posee el ser humano como todo y parte - de la sociedad- en el cual se relaciona su existencia; mientras que Nussbaum habla de capacidades combinadas mediante las cuales se produce la conjunción de las habilidades personales y las posibilidades que ofrece el entorno.

El fundamento último de la vida en sociedad para Ruiz Sánchez (1978a) consiste en:

por un lado la necesidad (indigencia) de bienes que no se tienen pero que son exigidos, indispensables, para una naturaleza que los busca y que viene estructuralmente preparada para encontrarlos pero es inicialmente inhábil para lograrlo. Por otro lado, la posibilidad de respuesta, por la inteligencia y la voluntad de otros, y, más tarde, por las propias. (p. 207)

En esta ayuda para la consecución de bienes que no se tienen se tejen las relaciones sociales, que también pueden ser afectadas por la falibilidad. De allí que la plenitud del ser humano exija la adecuada proyección de la conducta al bien común (Ruiz Sánchez, 1978a).

Por último, los fundamentos éticos constituyen el tercer gran pilar en el que se asienta la educación según Ruiz Sánchez. El autor mendocino parte de la necesidad de reconocer un fin en la naturaleza humana que el ser humano apetece. Por ello, desarrolla un breve tratado moral que da pie a su abordaje acerca de los fines de la educación.

Según lo expuesto por el autor en dicho tratado (Ruiz Sánchez, 1978a) se deduce que los fines de la educación están subordinados a los fines del ser humano y que estos parten de la concepción ética y antropológica que se adopte. El fin de la educación en Ruiz Sánchez es la plenitud dinámica. Esta incluye múltiples aspectos que desarrolla, y aquí solo nos limitamos a mencionar: la ordenación desde la interioridad y la proyección adecuada "ad extra", la formación o sabiduría práctica (constituida por la conciencia moral, verdadera, recta, cierta; la comprensión del sentido de la propia vida; la capacidad de juicio crítico objetivo; la docilidad o disposición de apertura espiritual y la mentalidad adecuada) y el orden interior (dentro del cual se encuentran la libertad, dominio o posesión de sí y ordenación del dinamismo interior interpotencial).

Este breve panorama permite comprender el concepto de educación que va a definir el autor, el cual sostiene que desde el ángulo del agente la educación es:

El auxilio al hombre, en tanto que indigente y falible, por el cual éste puede lograr su plenitud dinámica, esto es, la capacidad estable para ordenarse libre y rectamente, en su dinamismo interior y su autoconducción hacia los bienes individuales y comunes, naturales y sobrenaturales que plenifican su naturaleza. (Ruiz Sánchez, 1978a, p. 21) 
doi: http://doi.org/10.15359/ree.24-1.15

URL: http://www.una.ac.cr/educare

CORREO: educare@una.cr

La educación viene, entonces, a ser una ayuda para que el educando logre ese hábito que a modo de segunda naturaleza le permite obrar adecuadamente en todas sus dimensiones y relaciones sociales existentes. Consiste, por lo tanto, en una especie de actitud que favorece constantemente la plenificación del ser humano, aunque este siempre se halle susceptible de falibilidad.

\section{La necesidad de una nueva mirada humana: Diálogo entre la autora y el autor}

El contexto contemporáneo en el cual se mueve la educación requiere repensar, entre otras cosas, qué es el ser humano, pues la educación debe ser una tarea a su medida y no en orden a los intereses foráneos de las naciones. El enfoque de desarrollo humano o de las capacidades lleva a cuestionarnos en la actualidad el lugar del ser humano no como medio, sino como fin de las políticas sociales y la vinculación de estas con el reconocimiento de una vida plenamente humana.

El mérito de Nussbaum radica en pensar al ser humano por símismo, no en función de otros fines y elaborar una propuesta inversa a los intereses utilitarios, pues sostiene que la sociedad, el Estado y las políticas públicas deben disponerse en función del ser humano. Como bien advierte la autora (Nussbaum, 2010), la educación nos prepara no solo para la ciudadanía, sino también para el trabajo $y$, sobre todo, para darle sentido a nuestra vida. De aquí se desprende el sentido humano de la educación que intentamos revalorizar desde la mirada de Nussbaum. En efecto, Retomar el sentido que tiene la educación del ser humano de acuerdo con su dignidad y en dirección a su plenificación han sido los objetivos que han guiado este trabajo.

Por otro lado, conviene tener presente que Nussbaum $(2012$, p. 38) no deja de reparar en que "el enfoque proporciona una buena base para la edificación de una teoría de la justicia y los derechos". En efecto, este enfoque puede ser un medio para evaluar la calidad de vida y la justicia social y, en él mismo se puede vislumbrar un giro en cuanto a la visión antropológica, ya que aquí el ser humano constituye un fin en sí mismo y no un mero instrumento de la economía global.

En este sentido, Ruiz Sánchez (1972b, 1978a) enfatiza también la necesidad de un concepto de educación adecuado de ser humano, pues tratar de dilucidar cómo debe ser su educación y a qué apuntar en materia educativa; pues sin definir antes tal concepto es carecer de un marco teórico sólido a partir del cual se pueda establecer un rumbo coherente y eficaz. De allí que el sentido de la educación desde la mirada del profesor mendocino revitalice el carácter falible y perfectivo del ser humano, y propone una finalidad educativa a su medida, esto es, como plenitud dinámica. 


\section{Conclusiones}

En este breve esbozo se mostró que ambas propuestas -la de Nussbaum y Ruiz Sánchezestán centradas en la elección o libertad humana; además, están comprometidas con la autodefinición de las personas, de aquí que adquiere el sentido educativo desde ambas miradas cierta similitud. En efecto, la razón práctica puede ser entendida desde la plenitud dinámica, aunque en esta última se reconoce un contenido objetivo "perfectible" y, que, por tanto, le da plenitud al mismo ser humano. En Ruiz Sánchez, la educación ordena la interioridad y la conducta del individuo hacia los bienes que perfecciona su naturaleza. Si bien estos bienes se presentan de manera distinta según cada sujeto y el desenvolvimiento de su existencia, se deja ver un respeto hacia la objetividad, que proclama en la realidad un valor objetivo de los bienes que perfeccionan al ser humano; si el ser humano renuncia a dichos bienes deja al margen su plenitud, de allí la necesidad de lo que el autor denomina mentalidad adecuada y juicio crítico (Ruiz Sánchez, 1978a). Este último es una especie de valoración con respecto a la interioridad, las aptitudes, las inclinaciones, las limitaciones del sujeto y las conductas posibles en relación con sus fines perfectivos, y las circunstancias en las que está inmerso. En todos los sentidos, para Ruiz Sánchez los bienes a los que se tiende deben ser, necesariamente, perfectivos y la educación colabora en tal auxilio, por eso para ella el concepto de naturaleza humana es el sustrato primero.

Por el contrario, en Nussbaum puede concederse cierta autoridad al consenso y a la relatividad del bien por no estar sustentada su teoría desde un cimiento metafísico como ella misma ha sostenido (Nussbaum, 2007, 2012).

Puede observarse otra distinción fundamental entre ambos pensamientos en el hecho de que en Nussbaum hay una fuerte demanda de la presencia del Estado y de las políticas públicas para garantizar el desarrollo de las capacidades; mientras que, en Ruiz Sánchez, esto, de algún modo, está presente, pero figurado en la representación del bien común que se debe garantizar, por consiguiente, la propuesta de Nussbaum adquiere un grado mayor de concreción y determinación de la finalidad específica de la educación en pos de garantizar la igualdad social y el funcionamiento de las capacidades desde las políticas públicas. De este modo, la propuesta de la autora se acerca más a una filosofía política o sociología.

Desde otra perspectiva, Ruiz Sánchez centra su atención en los fundamentos y fines que motivan la educación en sí misma desde una visión antropológica, de allí que podamos comprenderlo como una propuesta que genera una idea del desarrollo humano o capacidades en un sentido más radical, en términos de una filosofía de la educación.

A su vez, puede encontrarse una similitud, si se considera que en el autor mendocino se advierte la posibilidad de plenificación según cada sujeto y también en constante dinamismo; esto puede asociarse a las capacidades combinadas que son la totalidad de las oportunidades 
doi: http://doi.org/10.15359/ree.24-1.15

URL: http://www.una.ac.cr/educare

CORREO: educare@una.cr

que se dispone para elegir y para actuar en cada situación concreta (Nussbaum, 2012) y que están relacionadas con estados fluidos y dinámicos, que representan en Nussbaum las capacidades internas (Nussbaum, 2012).

En suma, tanto Francisco Ruiz Sánchez como Martha Nussbaum han abordado esta histórica y constante búsqueda del desarrollo y la plenificación humana en orden a otorgarle un sentido netamente humano a la educación, que vaya más allá de los intereses utilitarios de las naciones.

Hemos intentado, en este escrito, interpretar cómo es el sentido de la educación desde lo humano desde dos miradas: la de Martha Nussbaum y Francisco Ruiz Sánchez quienes enfatizan la necesidad de una educación a la medida del ser humano, ya sea, desde la educación en el marco del desarrollo de capacidades o desde la educación como plenitud humana. Estimamos que ambas miradas apuestan una concepción humanista que dota de sentido la educación y trasciende los planteos mercantilistas que estrechan la visión educativa. En conclusión, tanto Nussbaum como Ruiz Sánchez apuestan a una comprensión educativa a la medida del ser humano.

\section{Referencias}

Arjona, G. E. (2013). Democracia y liberalismo político. La perspectiva de Martha Nussbaum. Colombia internacional, 78, 145-180. doi: https://doi.org/10.7440/colombiaint78.2013.06

Bicocca, M. (2008). Elementos a consideraren la metodología dela investigación filosófica. Síntesis. Revista de Filosofía, 2(1), 95-108. doi: https://doi.org/10.15691/0718-5448Vol2Iss1a68

Bicocca, M. (2015). Filosofía de la educación en Martha Nussbaum. Críticas al actual modelo mercantilista de la educación superior. Psicopedagógica, 13, 27-39.

Dewey, J. (1916). Democracy and education. New York: The Macmillan Company.

Gadamer, H.-G. (2015). Verdad y método II (9ºd.). Salamanca: Sígueme.

García-Amilburu, M. (2008). La cultura como texto. Hermenéutica y educación. En J. E. Ortega (ed.), Cultura, hermenéutica y educación (pp. 105-118). Valladolid: Servicio de Publicaciones de la Universidad Europea Miguel de Cervantes-CEINCE.

Gómez, Á. (2013). Ética del desarrollo humano según el enfoque de las capacidades de Matha Nussbaum. Phainomenon, 12(1), 19-28. Recuperado de http://revistas.unife.edu.pe/index. php/phainomenon/article/view/238/351

Gough, I. (2007-2008). El enfoque de las capacidades de M. Nussbaum: Un análisis comparado con nuestra teoría de las necesidades humanas (Trad. L. Nagore y S. Silva). Papeles de Relaciones Ecosociales y Cambio Global, 100, 177-202. Recuperado de http://otrodesarrollo. com/desarrollohumano/GoughEnfoqueCapacidadesNusbaum.pdf 
McCarthy, T. (1992). La teoría crítica de Jürgen Habermas. Madrid: Tecnos.

Monereo, C. (2015). Martha C. Nussbaum - Otro enfoque para la defensa del ser humano y de los derechos de las mujeres. Seqüência, 70, 93-114. doi: http://dx.doi.org/10.5007/21777055.2015v36n70p93

Murillo, F. J. y Hernández, R. (2011). Hacia un concepto de justicia social. Revista lberoamericana sobre Calidad, Eficacia y Cambio en Educación, 9(4), 7-23. Recuperado de http://www. redalyc.org/pdf/551/55122156002.pdf

Nussbaum,M.C.(1992).Humanfunctioningandsocialjustice:IndefenseofAristotelianEssentialism. Political Theory, 20(2), 202-246. doi: https://doi.org/10.1177/0090591792020002002

Nussbaum, M. C. (1995). Introducción: Forma y contenido, filosofía y literatura. Estudios de Filosofía, 11, 43-106.

Nussbaum, M.C. (2000). Aristotle, politics, and human capabilities: A response to Antony, Arneson, Charlesworth and Mulgan. Ethics, 111(1), 102-140. doi: https://doi.org/10.1086/233421

Nussbaum, M. C. (2007). Las fronteras de la justicia. Consideraciones sobre la exclusión. Barcelona: Paidós.

Nussbaum, M. C. (2010). Sin fines de lucro. Por qué la democracia necesita de las humanidades. Buenos Aires: Katz. doi: https://doi.org/10.2307/j.ctvndv60c

Nussbaum, M. C. (2012). Crear capacidades. Propuesta para el desarrollo humano. Barcelona: Paidós.

O'Brien, R. (2010). Prefacio. En Nussbaum, M. C. (Autora), Sin fines de lucro. Por qué la democracia necesita de las humanidades (pp. 11-14). Buenos Aires: Katz. doi: https://doi.org/10.2307/j. ctvndv60c.3

Pring, R. (2016). Una filosofía de la educación políticamente incómoda. Madrid: Narcea.

Ruiz Sánchez, F. (1962a). Perfectibilidad y educabilidad. Sapientia, 17(65), 178-192. Recuperado de http://bibliotecadigital.uca.edu.ar/greenstone/cgi-bin/library.cgi?a=d\&c=Revistas\&d= sapientia65

Ruiz Sánhez, F. (1962b). El concepto del hombre y la educación. Boletín de Estudios Políticos y Sociales, 12, 65-80.

Ruiz Sánchez, F. (1972a). Acerca de la educabilidad. Cuadernos de Pedagogía, 1, 1-65.

Ruiz Sánchez, F. (1972b). Pedagogía [Documento de cátedra organizado por estudiantes del profesor Francisco Ruiz Sánchez]. Mendoza: Universidad Católica Argentina. 
doi: http://doi.org/10.15359/ree.24-1.15

URL: http://www.una.ac.cr/educare

CORREO: educare@una.cr

Ruiz Sánchez, F. (1973a). Esquema tentativo para una estructuración de la temática fundamental de la pedagogía. Mendoza: Universidad Nacional De Cuyo, Instituto de Filosofía, Facultad de Filosofía y Letras.

Ruiz Sánchez, F. (1973b). Los fines de la educación. Cuadernos de Pedagogía, 2, 1-45.

Ruiz Sánchez, F. (1978a). Fundamentos y fines de la educación. Mendoza: Universidad Nacional de Cuyo, Facultad de Filosofía y Letras.

Ruiz Sánchez, F. (1978b). Introducción a los fundamentos antropológicos de la educación. Universidad Nacional de Entre Ríos (ed.), Actas de las Primeras Jornadas Nacionales Universitarias de Filosofía de la Educación (pp. 39-58). Paraná: Universidad Nacional de Entre Ríos.

Ruiz Sánchez, F. (1978c). Posibilidad de considerar la educación como un arte. Philosophica, 1, 219-233.

Ruiz Sánchez, F. (1978d). El hombre ¿actor o juguete de la historia? Mikael, 6(16), 59-77.

Ruiz Sánchez, F. (1979). La contaminación de la educación. En P. Randle (ed.), La educación ambiental (pp. 265-275). Buenos Aires: Oikos.

Sen, A. (2004). Capabilities, lists, and public reason: Continuing the conversation. Feminist Economics, 10(3), 77-80. doi: https://doi.org/10.1080/1354570042000315163 